\title{
Epidermal growth factor treatment has protective effects on the integrity of the blood-brain barrier against cerebral ischemia injury in bEnd3 cells
}

\author{
SHU YANG $^{1}$, HONG JIN ${ }^{2}$ and ZHI-GANG ZHAO ${ }^{1}$ \\ ${ }^{1}$ Department of Pharmacy, Beijing Tiantan Hospital, Capital Medical University, Beijing 100070; \\ ${ }^{2}$ Disinfection Evaluation Research Center, Institute of Disease Prevention and Control of PLA, Beijing 100071, P.R. China
}

Received July 27, 2018; Accepted December 31, 2018

DOI: $10.3892 /$ etm.2019.7186

\begin{abstract}
Tight junctions (TJs) serve an important role in maintaining the integrity of the blood-brain barrier (BBB), while neurological disorders, including ischemic stroke, induce TJ disruption and increase BBB permeability; results include edema formation and hemorrhage transformation. Cerebral endothelium protection presents a promising approach in ischemic stroke therapy. In the current study, protective effects of the epidermal growth factor (EGF) on ischemia-induced disruption of BBB integrity were examined using an oxygen-glucose deprivation (OGD) model in bEnd 3 cells. Expression levels of claudin-5 and TJ protein-1 (ZO-1) were determined by reverse transcription-quantitative polymerase chain reaction and western blot analysis. Cell viability was evaluated by cell counting kit- 8 assay and the endothelial permeability of Lucifer yellow (LY) was assessed using Transwell assays. The results revealed that post-ischemia administration of EGF $(250 \mathrm{ng} / \mathrm{ml})$ significantly attenuated the decrease in mRNA $(\mathrm{P}<0.05)$ and protein $(\mathrm{P}<0.01)$ expression levels of claudin-5 and ZO-1, and the increase in endothelial permeability of LY $(\mathrm{P}<0.05)$ induced by $4 \mathrm{~h}$ OGD exposure followed by $24 \mathrm{~h}$ reoxygenation. In addition, EGF did not significant affect cell viability. The current study suggested a potential of EGF to improve BBB integrity against ischemic injury by upregulating the expression of TJ proteins and reducing endothelial permeability.
\end{abstract}

\section{Introduction}

The blood-brain barrier (BBB) is a specialized tissue that restricts certain substances from entering the brain and

Correspondence to: Professor Zhi-Gang Zhao, Department of Pharmacy, Beijing Tiantan Hospital, Capital Medical University, 119 Nansihuan Xilu Road, Beijing 100070, P.R. China

E-mail: 1022zzg@sina.com

Key words: epidermal growth factor, blood-brain barrier, ischemia, tight junctions, bEnd3 protects normal neuronal function (1). The BBB is composed of cerebral endothelial cells, astrocytes and pericytes (2). Brain endothelial cells form the structural basis of the BBB and are characterized by the presence of tight junctions (TJs). TJs are composed of multiple protein complexes, including occludin, claudin-5 and TJ protein-1 (ZO-1) (3). Under normal conditions, TJs seal the gaps between endothelial cells and prevent the paracellular movement of molecules, wherein pathological insults caused by neurological disorders may lead to TJ disruption and BBB leakage, aggravating brain damage (4).

Ischemic stroke, which accounts for $\sim 85 \%$ of all cases of stroke, is one of the most common diseases worldwide with a high disability and mortality rates (5). The obstruction of blood vessels reduces oxygen and glucose supply to the cerebral tissue and results in an energy failure, which triggers a cascade of events, including the release of proinflammatory cytokines, elevated levels of reactive oxygen species (ROS) and the activation of matrix metalloproteinases (MMPs) (6). These lead to TJ protein degradation, which can be exacerbated by thrombolytic therapy (7). Enhanced BBB permeability induces the occurrence of deleterious complications, including brain edema and hemorrhage transformation (8). Numerous compounds, including $\alpha$-lipoic acid, ruscogenin and calycosin-7-O- $\beta$-D-glucoside, were reported to protect the ischemia-induced BBB injury through decreasing the generation of ROS or inhibiting the activity of MMPs (9-11). Therefore, targeting BBB integrity suggests a promising therapeutic approach for ischemic stroke treatment.

The epidermal growth factor (EGF) is a potent mitogen for mediating neuronal proliferation and inducing central nerve system (CNS) progenitor cells to produce astrocytes and neurons (12). EFG bioavailability in the brain is ensured through production in the CNS by glial cells and neurons and through uptake from the peripheral circulation (13). EGF has been examined as a neurotrophic factor against stroke in animal models in vivo (14). It also reduces BBB permeability by activating the EGF receptor and the downstream mitogen-activated protein kinase (MAPK) signaling pathway $(15,16)$. However, further investigation is required as to whether EGF may preserve BBB integrity against ischemic insult. 
In the current study, an in vitro oxygen-glucose deprivation (OGD) model was established based on bEnd 3 cells, to explore the benefit of EGF on BBB integrity and protection against ischemic injury. TJ protein expression levels were measured in addition to endothelial permeability and cell viability, to demonstrate the protective effect of EGF against ischemic injury by improving BBB integrity.

\section{Materials and methods}

Materials. bEnd3 cells were obtained from the American Type Culture Collection (Manassas, VA, USA). Dulbecco's modified Eagle's medium (DMEM), fetal bovine serum (FBS), penicillin-streptomycin solution and Hank's balanced salt solution (HBSS) were purchased from HyClone (GE Healthcare Life Sciences, Logan, UT, USA). Lucifer yellow (LY) was purchased from Sigma-Aldrich (Merck KGaA, Darmstadt, Germany). Rabbit claudin-5 antibody (cat. no. ab15106) was obtained from Abcam (Cambridge, UK). Rabbit ZO-1 antibody (cat. no. 21773-1-AP) was purchased from ProteinTech Group, Inc. (Chicago, IL, USA). Mouse GAPDH antibody (cat. no. C1312), horseradish peroxide (HRP)-conjugated goat anti-rabbit (cat. no. C1309) and anti-mouse (cat. no. C1308) IgG antibodies, the Cell Counting kit-8 (CCK8) and the bicinchoninic acid (BCA) assay kit were obtained from Applygen Technologies, Inc. (Beijing, China). Polyvinylidene difluoride (PVDF) membranes and the chemiluminescent HRP substrate (cat. no. WBKLS0100) were purchased from EMD Millipore (Billerica, MA, USA). The 24-well polyester Transwell inserts (pore size, $0.4 \mu \mathrm{M}$; diameter, $6.5 \mathrm{~mm}$ ) were obtained from Corning Inc. (Corning, NY, USA).

Cell culture and treatment.bEnd3 cells were cultured in DMEM supplemented with $10 \%$ FBS and $1 \%$ penicillin-streptomycin at $37^{\circ} \mathrm{C}$ with $5 \% \mathrm{CO}_{2}$ at a density of $3 \times 10^{5} /$ well in six-well plates. After $24 \mathrm{~h}$, confluent bEnd 3 cells were placed with glucose-free and serum-free DMEM (Beijing Solarbio Science \& Technology Co., Ltd., Beijing, China) and incubated in an anaerobic chamber $\left(1 \% \mathrm{O}_{2}\right)$ flushed with $95 \% \mathrm{~N}_{2}$ and $5 \% \mathrm{CO}_{2}$ for 4, 6 and $12 \mathrm{~h}$ (10). For reoxygenation (RO), cells were subsequently returned to normoxic conditions and incubated in glucose-containing and serum-free standard DMEM for $24 \mathrm{~h}$. For the control group, the bEnd 3 cells were incubated in glucose-containing and serum-free standard DMEM medium for the same period of time under normoxic conditions for the entire duration of the experiment. EGF-administered groups were treated with mouse EGF ( $250 \mathrm{ng} / \mathrm{ml}$; ProteinTech Group, Inc.) diluted with standard DMEM following a 4-h OGD period at the beginning of the RO process.

Cell viability. bEnd 3 cells $\left(5 \times 10^{3} /\right.$ well) were seeded in 96-well plates and incubated for $24 \mathrm{~h}$ prior to OGD treatment for 4,6 and $12 \mathrm{~h}$ as detailed above. A total of $10 \mu \mathrm{l}$ CCK8 solution was subsequently added to each well and incubated for $1 \mathrm{~h}$ at $37^{\circ} \mathrm{C}$. Absorbance was measured at $450 \mathrm{~nm}$ using a plate reader. Changes in cell viability were expressed compared with respective normoxic groups.

Western blot analysis. Cells were harvested following RO and homogenized in radioimmunoprecipitation assay buffer
(Applygen Technologies, Inc.) containing the protease inhibitor. The protein concentration was determined by BCA assays. Proteins $(40 \mu \mathrm{g})$ were separated on 12 and $8 \%$ SDS-PAGE gels for claudin-5 and ZO-1 analysis, respectively, and transferred to PVDF membranes. Following blocking with $5 \%$ non-fat milk for $1 \mathrm{~h}$ at room temperature, membranes were incubated with primary antibodies of claudin-5 (dilution, 1:1,000), ZO-1 (dilution, 1:1,000) and GAPDH (dilution, 1:2,000) at $4^{\circ} \mathrm{C}$ overnight, followed by incubation with secondary antibodies (dilution, 1:5,000) for $1 \mathrm{~h}$ at room temperature. Protein bands were visualized by chemiluminescence detection using a FluorChem FC2 system (Cell Biosciences, Inc., Santa Clara, CA, USA) and band densities were quantified by Image $\mathrm{J}$ (version 1.43; National Institute of Health, Bethesda, MD, USA). Bands were normalized to GAPDH and expressed in comparison with respective normoxic controls.

Reverse transcription-quantitative polymerase chain reaction (RT-qPCR) analysis. Total RNA was extracted from bEnd3 cells following RO using TRIzol reagent (Invitrogen; Thermo Fisher Scientific, Inc., Waltham, MA, USA) according to the manufacturer's protocol and quantified using a spectrophotometer. RNA $(1 \mu \mathrm{g})$ was reverse-transcribed into cDNA using the PrimeScript ${ }^{\mathrm{TM}}$ RT reagent kit with gDNA Eraser (Takara Bio, Inc., Otsu, Japan) according to the manufacturer's protocol. qPCR was performed using the SYBR Premix Ex Taq II kit (Takara Bio, Inc.) according to the manufacturer's protocol using the following thermocycling conditions: $95^{\circ} \mathrm{C}$ for $30 \mathrm{sec}$, followed by 40 cycles of $95^{\circ} \mathrm{C}$ for $5 \mathrm{sec}, 60^{\circ} \mathrm{C}$ for $20 \mathrm{sec}$ and $72^{\circ} \mathrm{C}$ for $30 \mathrm{sec}$. Primer sequences were as follows: Claudin-5, forward, 5'-GTTAAGGCACGGGTAGCACT-3' and reverse, 5'-TACTTCTGTGACACCGGCAC-3'; ZO-1, forward, 5'-AAGTTGGCAAGAGAGGAGCC-3' and reverse, 5'-CAACCGCATTTGGCGTTACA-3'; GAPDH, forward, 5'-ACGTCTGCCACGATAACACC-3' and reverse, 5'-CTG CATGATTGGGTCACGTC-3. GAPDH was used as internal control and mRNA expression levels were quantified using the $2^{-\Delta \Delta \mathrm{Cq}}$ method (17).

Permeability assay. bEnd3 cells (3.3x104/well) were seeded in Transwell inserts and incubated for 5 days at $37^{\circ} \mathrm{C}$ prior to performing the OGD. Following $4 \mathrm{~h}$ of OGD and $24 \mathrm{~h}$ of RO treatment, LY $(50 \mu \mathrm{M})$ dissolved in HBSS was added to the upper compartment and the lower compartment contained blank HBSS. At indicated time points (15, 30, 45 and $60 \mathrm{~min})$, samples were taken from the lower compartment and analyzed using a plate reader (excitation, $430 \mathrm{nM}$; emission, $540 \mathrm{nM}$ ). The permeability coefficient $\left(\mathrm{P}_{\mathrm{app}}\right)$ was measured by the following equation: $\mathrm{P}_{\mathrm{app}}=\mathrm{A} /\left(\mathrm{SxC}_{0}\right)$, where $\mathrm{A}$ represents the rate of drug accumulated at the lower compartment, $\mathrm{S}$ represents the membrane area and $\mathrm{C}_{0}$ is the initial drug concentration at the upper compartment.

Statistical analysis. Data are expressed as the mean \pm standard deviation, representative of three replicates. Differences between groups were assessed by one-way analysis of variance followed by Tukey's post hoc test using SPSS (version 19.0; IBM Corp., Armonk, NY, USA). P<0.05 was considered to indicate a statistically significant difference. 
A
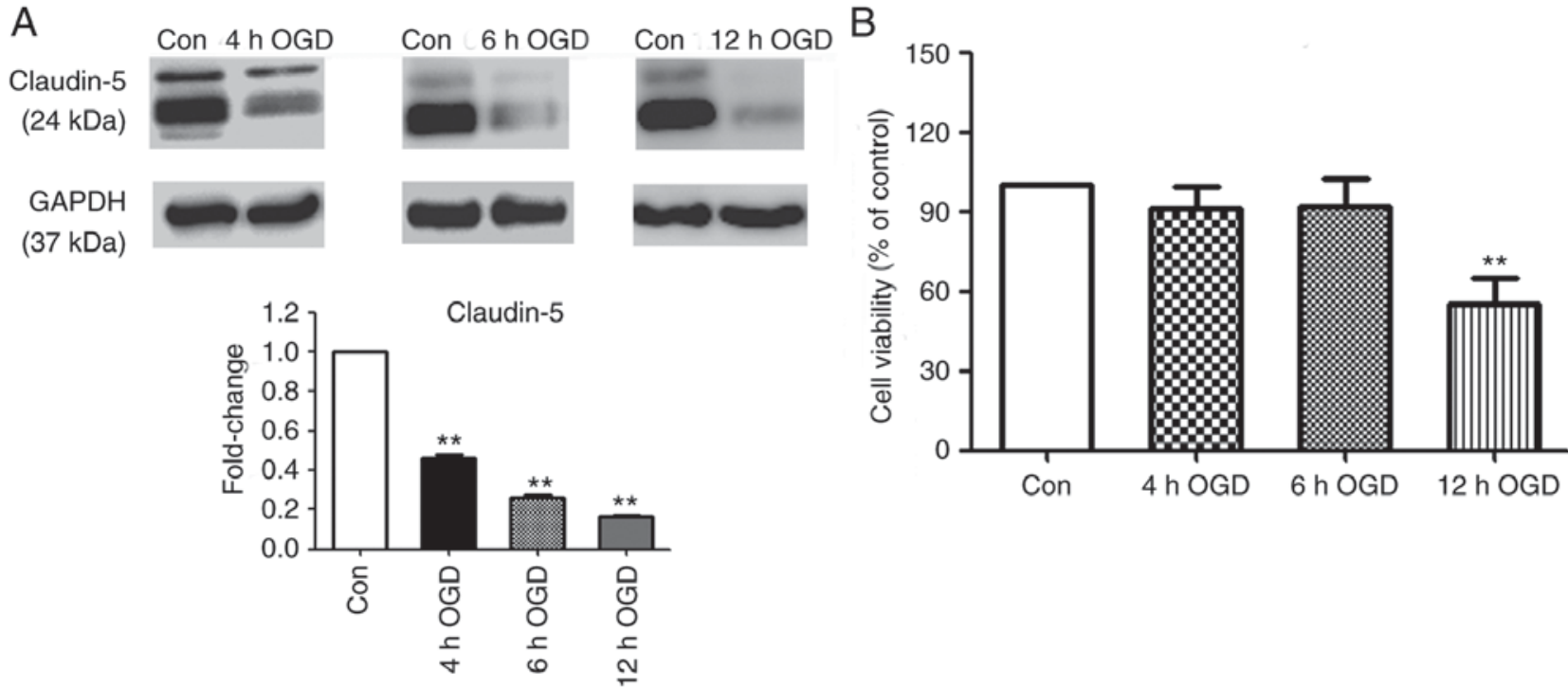

Figure 1. Effects of OGD on claudin-5 protein expression and cell viability in bEnd 3 cells. Cells were exposed to 4,6 and 12 h of OGD followed by 24 h reoxygenation. (A) Claudin- 5 protein expression measured by western blot analysis. (B) Cell viability determined by cell counting kit- 8 assays. ${ }^{* *} \mathrm{P}<0.01$ vs. Con. ODG, oxygen-glucose deprivation; Con, normoxic control.

\section{Results}

Effects of $O G D$ on TJ protein expression and cell viability. Claudin-5 is a key TJ protein that determines BBB permeability and integrity (18). As an indicator of ischemia-induced BBB disruption, claudin-5 expression was measured in bEnd3 cells, following exposure to OGD for various periods and $24 \mathrm{~h}$ RO. As presented in Fig. 1A, 4, 6 and 12 h OGD exposure resulted in a time-dependent decrease in claudin-5 protein expression compared with the normoxic controls. Cell viability analysis revealed no significant difference between the control and treatment groups following 4 and 6 h OGD exposure. In contrast, 12 h OGD exposure revealed a significant reduction of $45 \%$ in cell viability compared with the normoxic control $(\mathrm{P}<0.01$; Fig. 1B).

EGF attenuates $O G D$-induced BBB disruption. To identify protective effects of EGF on ischemia-induced BBB disruption, EGF was added to bEnd3 cells immediately following $4 \mathrm{~h}$ OGD exposure prior to RO and subsequently TJ protein expression levels were measured. In addition, endothelial cell permeability of LY and cell viability were determined. As presented in Fig. 2A and B, 4 h OGD exposure caused a significant decrease in claudin-5 and ZO-1 protein expression compared with the control (71.3 and 41.2\%, respectively; $\mathrm{P}<0.01$ ), which was significantly ameliorated by EGF treatment $(\mathrm{P}<0.01$ vs. OGD). However, the differences in the decrease of claudin-5 levels between the same groups in Fig. 1 (54.2\%) and Fig. $2(71.3 \%)$ may be attributed to the differential cell growth status and density prior to performing OGD in separate experiments. A significant decrease in mRNA expression by 61.5 and $52.1 \%$ was observed for claudin-5 and ZO-1 in the OGD group compared with the control group $(\mathrm{P}<0.01)$, while EGF significantly reversed this decrease $(\mathrm{P}<0.05$; Fig. $2 \mathrm{C})$. In addition, EGF treatment significantly attenuated an increase in endothelial permeability of LY following $4 \mathrm{~h}$ OGD exposure $(\mathrm{P}<0.05$ vs. OGD; Fig. 2D). No significant differences were observed in cell viability following EGF treatment compared with the OGD and normoxic control groups ( $\mathrm{P}>0.05$; Fig. 2E). Results indicated that the protective effect of EGF on BBB integrity may be via enhancing $\mathrm{TJ}$ protein expression and decreasing BBB permeability.

\section{Discussion}

Cerebral endothelial cells are a crucial component of the neurovascular unit and are implicated in many biological functions, including regulating vascular tone, providing trophic support for neurons and mediating inflammatory responses (9). They also contribute to BBB integrity by forming TJs, which block the paracellular flux (19). However, neurological disorders, including ischemic stroke, damage the cerebral endothelium leading to TJ dysfunction and BBB leakage, which are associated with complications, including brain edema and hemorrhage transformation (20). Therefore, there is an increasing interest in the protection of BBB integrity in stroke therapy (21).

The current study established an in vitro OGD model using the mouse brain microvascular endothelial cell line bEnd3 to mimic clinical ischemic stroke $(22,23)$. An increasing OGD exposure duration was observed to be associated with a decrease in claudin-5 protein expression, indicating a potential ischemia-induced BBB disruption. A 4 h OGD exposure was selected for subsequent studies as it closely reflected the limited therapeutic window of $4.5 \mathrm{~h}$ in ischemic stroke in a clinical setting (6). However, in contrast to previous studies, this duration of OGD exposure had no significant effect on cell viability. This may be attributed to the difference in oxygen levels during the OGD period, $1 \%$ here compared with $0.3 \%$ previously reported (24), which resulted in varying cell damage in the RO period.

The BBB phenotype of cerebral endothelial cells is regulated by the release of cytokines in the microenvironment (25). Glial-derived neurotrophic factor and basic 
A

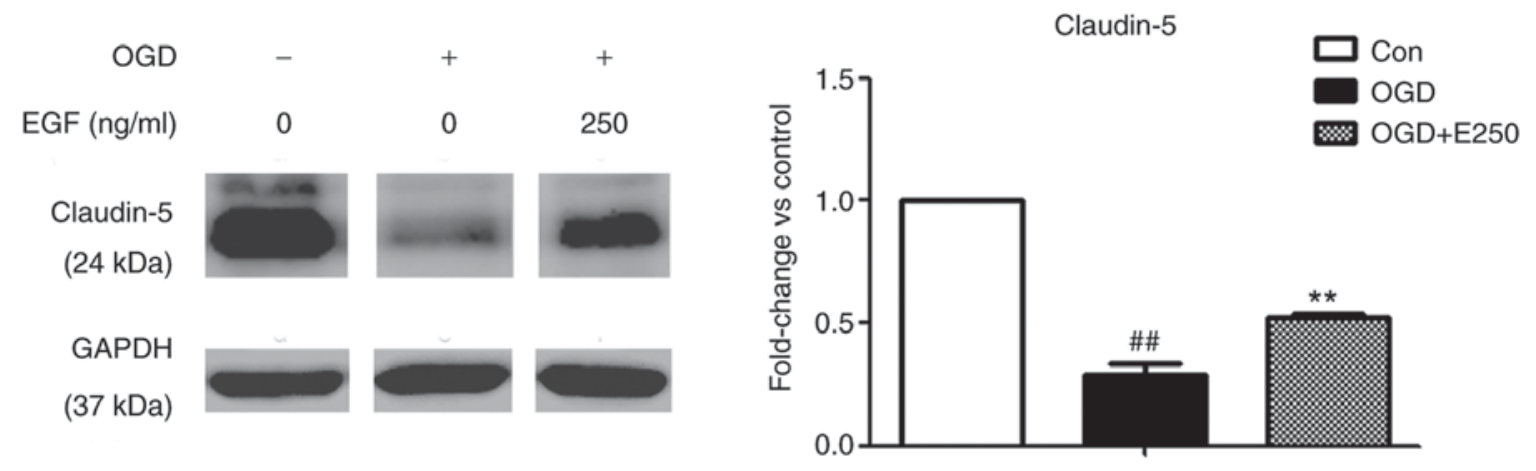

B
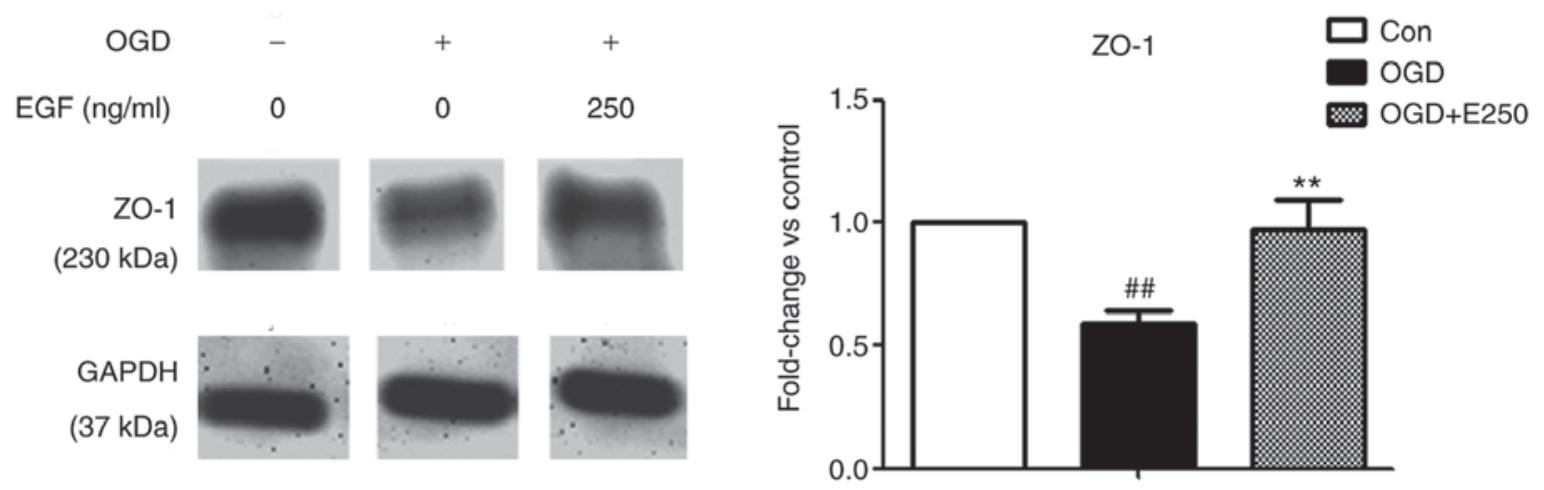

C

Claudin-5
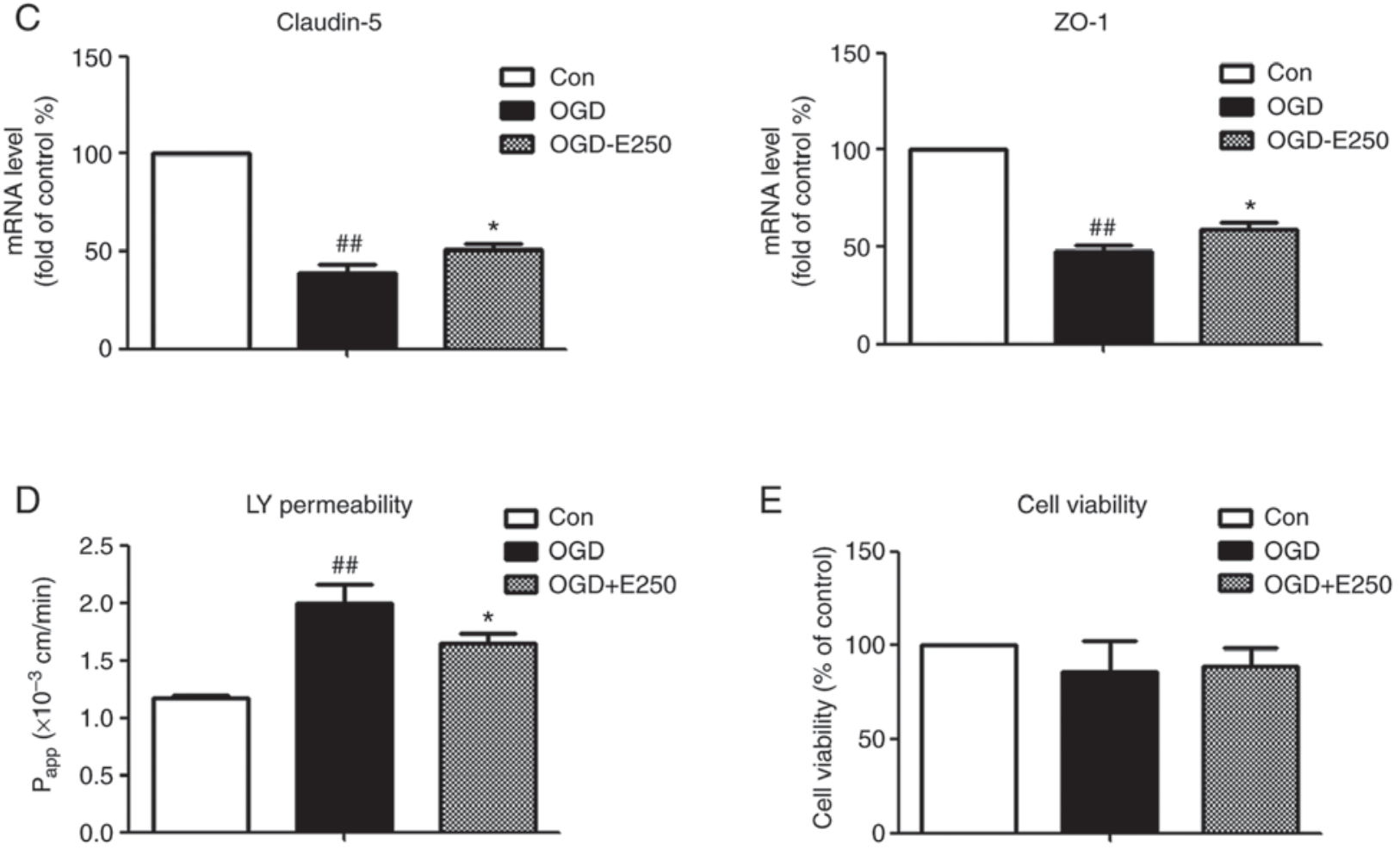

Figure 2. Effects of EGF on OGD-induced blood-brain barrier disruption in vitro. bEnd 3 cells were exposed to OGD for $4 \mathrm{~h}$ and treated with EGF (250 $\mathrm{ng} / \mathrm{ml}$ ) prior to $24 \mathrm{~h}$ reoxygenation. Protein expression of (A) claudin-5 and (B) ZO-1 was determined by western blotting. (C) mRNA expression levels of claudin-5 and ZO-1 were evaluated using reverse transcription-quantitative polymerase chain reaction assays. (D) Endothelial permeability of LY was determined by Transwell assay and (E) cell viability was evaluated using cell counting kit- 8 analysis. ${ }^{\# \prime} \mathrm{P}<0.01$ vs. Con; ${ }^{P}<0.05$ and ${ }^{* *} \mathrm{P}<0.01$ vs. OGD. EGF, epidermal growth factor; ODG, oxygen-glucose deprivation; Con, normoxic control; LY, Lucifer yellow; E250, $250 \mathrm{ng} / \mathrm{ml}$ EGF treatment; $P_{\text {app }}$, permeability coefficient.

fibroblast growth factor enhance TJ function, while proinflammatory cytokines, including interleukin-6 and tumor necrosis factor- $\alpha$ may damage BBB integrity (26). EGF has been reported to preserve endothelial integrity and protect
TJ disruption against hydrogen peroxide (27). In the present study it was demonstrated that EGF upregulated the mRNA and protein expression of claudin-5 and ZO-1 and reduced the endothelial cell permeability of LY against ischemic insults 
in vitro. As OGD and EGF exerted no significant influence on cell viability, according to the conditions evaluated in the present study, the protective effect of EGF on BBB integrity was mainly attributed to the elevation in TJ protein expression levels. Furthermore, MAPK and protein kinase B (Akt) signaling, downstream pathways of EGF, serve important roles in cell proliferation, survival and apoptosis (28). Their activation participates in the regulation of TJ protein expression and TJ function (29-31). Therefore, further investigation on whether EGF preserves BBB integrity against ischemic stroke by modulating the MAPK and Akt signaling pathways is required. Additionally, electron microscopy is an effective approach for observing $\mathrm{BBB}$ disruption at the ultrastructure level (32) and this method maybe explored in future BBB studies.

In summary, EGF ameliorated TJ protein degradation and inhibited increased BBB permeability induced by OGD treatment in bEnd 3 cells. The aforementioned findings indicated a beneficial effect of EGF on BBB integrity against ischemic insult.

\section{Acknowledgements}

Not applicable.

\section{Funding}

The present study was supported by the National Natural Science Foundation of China (grant no. 81703376) and the Youth Foundation of Beijing Tiantan Hospital (grant no. 2016-YQN-11).

\section{Availability of data and materials}

The datasets used and/or analyzed during the present study are available from the corresponding author on reasonable request.

\section{Authors' contributions}

SY and ZZ designed the study. SY performed all experiments. $\mathrm{SY}$ and $\mathrm{HJ}$ interpreted the data and drafted the manuscript. ZZ critically reviewed and revised the manuscript.

\section{Ethics approval and consent to participate}

Not applicable.

\section{Patient consent for publication}

Not applicable.

\section{Competing interests}

The authors declare that they have no competing interests.

\section{References}

1. Yang S, Mei S, Jin H, Zhu B, Tian Y, Huo J, Cui X, Guo A and Zhao Z: Identification of two immortalized cell lines, ECV304 and bEnd3, for in vitro permeability studies of blood-brain barrier. PLoS One 12: e0187017, 2017.
2. Cecchelli R, Berezowski V, Lundquist S, Culot M, Renftel M, Dehouck MP and Fenart L: Modelling of the blood-brain barrier in drug discovery and development. Nat Rev Drug Discov 6: 650-661, 2007.

3. Cardoso FL, Brites D and Brito MA: Looking at the blood-brain barrier: Molecular anatomy and possible investigation approaches. Brain Res Rev 64: 328-363, 2010.

4. Ronaldson PT and Davis TP: Blood-brain barrier integrity and glial support: Mechanisms that can be targeted for novel therapeutic approaches in stroke. Curr Pharm Des 18: 3624-3644, 2012.

5. Liu X, Zhu X, Chen M, Ge Q, Shen Y and Pan S: Resveratrol protects PC12 cells against OGD/R-induced apoptosis via the mitochondrial-mediated signaling pathway. Acta Biochim Biophys Sin (Shanghai) 48: 342-353, 2016.

6. Khatri R, McKinney AM, Swenson B and Janardhan V: Blood-brain barrier, reperfusion injury, and hemorrhagic transformation in acute ischemic stroke. Neurology 79 (13 Suppl 1): S52-S57, 2012.

7. Warach $\mathrm{S}$ and Latour LL: Evidence of reperfusion injury, exacerbated by thrombolytic therapy, in human focal brain ischemia using a novel imaging marker of early blood-brain barrier disruption. Stroke 35 (11 Suppl 1): S2659-S2661, 2004.

8. Zhu H, Wang Z, Xing Y, Gao Y, Ma T, Lou L, Lou J, Gao Y, Wang S and Wang Y: Baicalin reduces the permeability of the blood-brain barrier during hypoxia in vitro by increasing the expression of tight junction proteins in brain microvascular endothelial cells. J Ethnopharmacol 141: 714-720, 2012.

9. Xie R, Li X,Ling Y, Shen C, Wu X, Xu W and Gao X: Alpha-lipoic acid pre- and post-treatments provide protection against in vitro ischemia-reperfusion injury in cerebral endothelial cells via Akt/mTOR signaling. Brain Res 1482: 81-90, 2012.

10. Cao G, Jiang N, Hu Y, Zhang Y, Wang G, Yin M, Ma X, Zhou K, Qi J, Yu B and Kou J: Ruscogenin attenuates cerebral ischemia-induced blood-brain barrier dysfunction by suppressing TXNIP/NLRP3 inflammasome activation and the MAPK pathway. Int J Mol Sci 17: pii: E1418, 2016.

11. Fu S, Gu Y, Jiang JQ, Chen $X, X u ~ M$, Chen $X$ and Shen J: Calycosin-7-O- $\beta$-D-glucoside regulates nitric oxide/caveolin-1/matrix metalloproteinases pathway and protects blood-brain barrier integrity in experimental cerebral ischemia-reperfusion injury. J Ethnopharmacol 155: 692-701, 2014.

12. Pillai DR, Shanbhag NC, Dittmar MS, Boqdahn U and Schlachetzki F: Neurovascular protection by targeting early blood-brain barrier disruption with neurotrophic factors after ischemia-reperfusion in rats*. J Cereb Blood Flow Metab 33: 557-566, 2013.

13. Plata-Salamán CR: Epidermal growth factor and the nervous system. Peptides 12: 653-663, 1991.

14. Wang YF, Cooke MJ, Sachewsky N, Morshead CM and Shoichet MS: Bioengineered sequential growth factor delivery stimulates brain tissue regeneration after stroke. J Control Release 172: 1-11, 2013.

15. Liu W, Wang P, Shang C, Chen L, Cai H, Ma J, Yao Y, Shang X and Xue Y: Endophilin-1 regulates blood-brain barrier permeability by controlling $\mathrm{ZO}-1$ and occludin expression via the EGFR-ERK1/2 pathway. Brain Res 1573: 17-26, 2014.

16. Chen L, Liu W, Wang P, Xue Y, Su Q, Zeng C and Shang X: Endophilin-1 regulates blood-brain barrier permeability via EGFR-JNK signaling pathway. Brain Res 1606: 44-53, 2015.

17. Livak KJ and Schmittgen TD: Analysis of relative gene expression data using real-time quantitative PCR and the 2(-Delta Delta $\mathrm{C}(\mathrm{T}))$ method. Methods 25: 402-408, 2001.

18. Ronaldson PT and Davis TP: Targeting blood-brain barrier changes during inflammatory pain: An opportunity for optimizing CNS drug delivery. Ther Deliv 2: 1015-1041, 2011

19. Wilhelm I and Krizbai IA: In vitro models of the blood-brain barrier for the study of drug delivery to the brain. Mol Pharm 11: 1949-1963, 2014.

20. Choi KH, Kim HS, Park MS, Kim JT, Kim JH, Cho KA, Lee MC, Lee HJ and Cho KH: Regulation of caveolin-1 expression determines early brain edema after experimental focal cerebral ischemia. Stroke 47: 1336-1343, 2016.

21. Guo J, Krause DN, Horne J, Weiss JH, Li X and Duckles SP: Estrogen-receptor-mediated protection of cerebral endothelial cell viability and mitochondrial function after ischemic insult in vitro. J Cereb Blood Flow Metab 30: 545-554, 2010.

22. Liu J, Jin X, Liu KJ and Liu W: Matrix metalloproteinase-2-mediated occludin degradation and caveolin-1-mediated claudin-5 redistribution contribute to blood-brain barrier damage in early ischemic stroke stage. J Neurosci 32: 3044-3057, 2012. 
23. Rahman NA, Rasil ANHM, Meyding-Lamade U, Craemer EM, Diah S, Tuah AA and Muharram SH: Immortalized endothelial cell lines for in vitro blood-brain barrier models: A systematic review. Brain Res 1642: 532-545, 2016.

24. Ku JM, Taher M, Chin KY, Grace M, McIntyre P and Miller AA: Characterization of a mouse cerebral microvascular endothelial cell line (bEnd.3) after oxygen glucose deprivation and reoxygenation. Clin Exp Pharmacol Physiol 43: 777-786, 2016.

25. Igarashi $Y$, Utsumi $H$, Chiba $H$, Yamada-Sasamori $Y$, Tobioka $H$ Kamimura Y, Furuuchi K, Kokai Y, Nakagawa T, Mori M and Sawada N: Glial cell line-derived neurotrophic factor induces barrier function of endothelial cells forming the blood-brain barrier. Biochem Biophys Res Commun 261: 108-112, 1999.

26. Abbott NJ, Rönnbäck L and Hansson E: Astrocyte-endothelia interactions at the blood-brain barrier. Nat Rev Neurosci 7: 41-53, 2006.

27. Basuroy S, Seth A, Elias B, Naren AP and Rao R: MAPK interacts with occludin and mediates EGF-induced prevention of tight junction disruption by hydrogen peroxide. Biochem J 393: 69-77, 2006.
28. Park $\mathrm{JH}$ and Han HJ: Caveolin-1 plays important role in EGF-induced migration and proliferation of mouse embryonic stem cells: Involvement of PI3K/Akt and ERK. Am J Physiol Cell Physiol 297: C935-C944, 2009.

29. Campbell M, Collery R, McEvoy A, Gardiner TA, Stitt AW and Brankin B: Involvement of MAPKs in endostatin-mediated regulation of blood-retinal barrier function. Curr Eye Res 31: 1033-1045, 2006

30. Sumanasekera WK, Sumanasekera GU, Mattingly KA, Dougherty SM, Keynton RS and Klinge CM: Estradiol and dihydrotestosterone regulate endothelial cell barrier function after hypergravity-induced alterations in MAPK activity. Am J Physiol Cell Physiol 293: C566-C573, 2007.

31. Luo D, Zhao J and Rong J: Plant-derived triterpene celastrol ameliorates oxygen glucose deprivation-induced disruption of endothelial barrier assembly via inducing tight junction proteins. Phytomedicine 23: 1621-1628, 2016.

32. Lu D, Mai HC, Liang YB, Xu BD, Xu AD and Zhang YS: Beneficial role of rosuvastatin in blood-brain barrier damage following experimental ischemic stroke. Front Pharmacol 9: 926, 2018. 